\section{Actualización en depresión postparto: el desafío permanente de optimizar su detección y abordaje}

\author{
CONSTANZA MENDOZA B. ${ }^{a}$, SANDRA SALDIVIA ${ }^{\mathrm{b}}$
}

\section{An update on postpartum depression}

Postpartum depression (PPD) is a common condition worldwide and most of the available information is about treatment rather than prevention. This paper is an update on prevention and treatment of PPD. A simple review of the literature and a critical review of papers' methodology and conclusions was carried out. There is a consensus of the preeminence of psychosocial factors in the genesis of PPD. Considering the complications when the condition is not treated, it is of the utmost importance to implement early detection and management strategies. The use of psychosocial preventive interventions is an alternative that has support in the literature and should be seriously considered.

(Rev Med Chile 2015; 143: 887-894)

Key words: Depression Postpartum; Prevention; Risk factors; Therapeutics.
Departamento de Psiquiatría y Salud Mental. Facultad de Medicina. Universidad de Concepción. Concepción, Chile. aPsiquiatra. Doctorada en Salud Mental.

bPsicóloga. Doctora (PhD.) en Psicología.

Financiamiento: Proyecto de la Universidad de Concepción № 213.087.042-1.0.

Recibido el 24 de julio de 2014, aceptado el 26 de abril de 2015.

Correspondencia a:

Constanza Mendoza Bermúdez 5 de Abril № 570.

Chillán, VIII Región, Chile.

Teléfono-fax: 042-2432911.

constanzamendozab@gmail.com

\section{L} a depresión postparto (DPP) es la complicación psiquiátrica más frecuente del postnatal y, dadas sus potenciales repercusiones en el bienestar de la madre y del bebé, su correcto abordaje debe constituir una prioridad en Salud Pública. Además, provee una especial oportunidad de prevención pues está precedida por un "marcador" definido: el nacimiento de un hijo. Se han identificado grupos de riesgo y un período de mayor vulnerabilidad, que son los primeros tres meses del período postnatal ${ }^{1}$. El Manual Diagnóstico y Estadístico de Trastornos Mentales en su quinta edición (DSM-V) ${ }^{2}$ incluyó como novedad frente a la versión anterior ${ }^{3}$, el trastorno depresivo mayor de inicio en el periparto, considerando su desarrollo durante el embarazo o en las primeras cuatro semanas del postparto. En este punto mantuvo el mismo criterio de tiempo, lo que ha generado cuestionamientos, ya que, para efectos clínicos y de investigación, el período de riesgo puede ampliarse a los tres meses del parto ${ }^{4}$.

La prevalencia mundial de DPP a lo largo de la vida fluctúa entre 10 y $20 \%{ }^{4,5}$, observándose mayores valores en madres adolescentes $(26 \%)^{6}$ y en los niveles socioeconómicos más bajos
$(38,2 \%)^{7}$. Un estudio antropológico publicado a fines de la década 1980-89 sugirió que la DPP era prácticamente inexistente en China, debido a una mayor atención dirigida a la mujer y a las adecuadas redes de apoyo familiar. Estudios posteriores con instrumentos de auto-reporte han arrojado tasas entre 0 y $18 \%$ en poblaciones diversas de ese país ${ }^{8}$. Respecto de su prevalencia en el mundo occidental, se han reportado valores más altos en países emergentes que en los desarrollados ${ }^{9}$. Sin embargo, la evaluación de algunos estudios epidemiológicos en DPP no es fácil debido a las variaciones en sus diseños metodológicos (ej. uso de instrumentos de tamizaje vs detección espontánea de los síntomas), en los tamaños muestrales y en los criterios diagnósticos utilizados.

En Chile, Alvarado y cols. $(1992)^{10}$ reportaron una incidencia de $8,8 \%$, y una prevalencia de $20,5 \%$, y Jadresic y cols. $(1992)^{11}$ de $9,2 \%$ y $10,2 \%$, respectivamente. Jadresic y Araya (1995) $)^{12}$ observaron una relación inversa entre su prevalencia y el nivel socioeconómico, alcanzando $41,3 \%$ en el bajo, $33,5 \%$ en el medio, y $27,7 \%$ en el alto. Es decir, las mujeres con menores ingresos exhibieron aproximadamente el triple del riesgo 
(OR 2,7; IC 95\%; [1,4-5,2]; p = 0,00). La mayoría de las mujeres afectadas puede tener síntomas durante seis meses y $25 \%$ de ellas por un año, si no reciben tratamiento ${ }^{13}$. El riesgo de recurrencia de DPP también se aproxima a $25 \%{ }^{1}$, y aumenta la probabilidad de depresión no ligada al postparto, especialmente cuando existen antecedentes personales de este tipo ${ }^{14}$.

El objetivo del presente artículo es realizar una revisión simple de la información vigente sobre la DPP, con mayor énfasis en los factores causales, los desafíos en el diagnóstico y su adecuado abordaje.

\section{Metodología}

Realizamos una búsqueda de información en español e inglés en las siguientes bases electrónicas: Medline, Elsevier, ISI Web of Science, ProQuest y Cochrane Library, durante el segundo semestre de 2014; como "palabras clave" se incluyeron: depresión postparto (postpartum depresion), factores de riesgo (risk factors), hormonas (hormones), prevención (prevention), tratamiento (treatment). Se seleccionaron un total de 53 referencias conformadas por: un protocolo nacional, una guía clínica nacional, dos manuales internacionales, cuatro revisiones, seis metaanálisis, y 39 artículos originales, publicados en los últimos 25 años. Los criterios de inclusión de los artículos se fundamentaron en la metodología empleada, sus resultados, la relevancia de las publicaciones y en la experiencia de los autores en el estudio de la DPP; las otras referencias (protocolos, guías, y manuales) se eligieron por ser el resultado de un consenso de expertos a nivel nacional e internacional.

\section{Resultados}

A continuación revisaremos las 39 referencias restantes, distribuidas en: 25 artículos, un protocolo y una guía clínica, para abordar el diagnóstico y detección de predictores psicosociales y, 12 artículos referentes al abordaje de la DPP.

\section{Aspectos clínicos y diagnóstico}

Aproximadamente 60 a $85 \%$ de mujeres puede presentar algunos síntomas de apariencia depresiva en los primeros días del postparto. Generalmente estos son leves y se resuelven pronto, lo que se conoce como "postpartum blues" o disforia postparto $^{15}$. En contraste, la DPP consta de la sintomatología propia de cualquier episodio depresivo mayor y puede acompañarse de ideas obsesivas respecto del bienestar del recién nacido ${ }^{15,16}$. Puede presentarse ansiedad, pobre percepción del rol materno, y mayores alteraciones motoras o cognitivas en comparación con las depresiones no ligadas al postparto ${ }^{17}$. Lo anterior debe ocurrir la mayor parte del tiempo durante dos semanas, afectando el funcionamiento cotidiano y el cuidado del bebé ${ }^{15,18}$. Los desenlaces más temidos incluyen el suicidio e infanticidio, que habitualmente están más asociados a la ausencia de tratamiento, DPP severa y psicosis postparto. Sin embargo, Paris y cols. $(2009)^{17}$ evaluaron a 32 mujeres con DPP, encontrando que $43 \%$ presentaba "moderado a alto riesgo suicida", asociado significativamente con una baja autoestima en relación a la interrupción de su trabajo, mayor estrés en el rol materno, menor preparación para asumirlo, e inadecuada interacción con su bebé. Además, la duración de un episodio depresivo postparto afecta de forma proporcional el vínculo materno-filial, generando un impacto negativo en el desarrollo emocional y cognitivo del bebé, potenciando los efectos de variables asociadas con deprivación mental como el bajo nivel socioeconómico ${ }^{19}$. Los hijos de madres con DPP pueden establecer relaciones de "apego ansioso" con una frecuencia significativamente mayor que los hijos de madres no deprimidas, y exhibir una mayor probabilidad de trastornos conductuales, accidentes, ingresos hospitalarios y maltrato infantil ${ }^{19}$.

También se ha observado que la sintomatología depresiva persistente de la mujer en el postnatal interfiere con el bienestar de la familia e, incluso, aumentaría la probabilidad de depresión en su pareja ante la presencia de otros factores como: baja escolaridad, pobre apoyo psicosocial, y violencia en la relación marital ${ }^{20}$.

Un tema relevante respecto del pronóstico de la DPP es la alta probabilidad de sub-diagnóstico, que puede vincularse con: 1) Una mayor atención al bienestar del bebé en este período; 2) para la mujer es difícil asumir y priorizar su condición emocional y 3) el estigma, la vergüenza, y el temor a la crítica ${ }^{15}$. Por lo tanto, es importante que el personal de salud evalúe durante el embarazo la presencia de síntomas psicológicos depresivos, pues algunas gestantes pueden cursar con fatiga, labilidad emocional, alteraciones del sueño o del apetito ${ }^{16}$, lo que "encubriría" el diagnóstico. 
La pesquisa sistemática de estos síntomas debe continuar durante el postparto, especialmente en mujeres con factores de riesgo o sintomatología antenatal ${ }^{15}$.

En Chile, el Ministerio de Salud ha recomendado el uso de la Escala de Depresión Postparto de Edimburgo (EDPE) como instrumento de tamizaje de la depresión en el embarazo y postparto ${ }^{15}$, la cual se encuentra validada en mujeres chilenas desde $1995^{21}$. En el embarazo se indicó su aplicación en el primer o segundo control gestacional, y un puntaje de corte de 13 determina sospecha de depresión. En el postparto, el puntaje de corte es 11 puntos, aconsejando su medición a las ocho semanas, y al sexto mes ${ }^{15}$. En 2008, Castañón y Pinto ${ }^{22}$ reportaron una tasa de detección espontánea de síntomas depresivos postparto de $4,6 \%$, que alcanzó $26,4 \%$ usando la EDPE con un puntaje de corte de 10. Además, encontraron una incidencia de 15\% de DPP usando la Entrevista Clínica Estructurada para los Trastornos del Eje I del DSM-IV (SCID-I). Sus resultados son semejantes al estudio de Evins y cols. $(2000)^{23}$ que también destaca la utilidad de la EDPE en la adecuada detección de síntomas depresivos perinatales.

\section{Factores causales}

A diferencia de otros trastornos psiquiátricos, del postparto como la psicosis puerperal, que ha mostrado una mayor relación con aspectos biológicos, los factores que han mostrado una mayor asociación con la DPP son de tipo psicosocial2 ${ }^{24}$. Basados en los resultados de varios metaanálisis publicados desde 1996 al 2004 $25-27$, estos predictores se han clasificado, de acuerdo al tamaño del efecto, en grande a moderado, moderado, y pequeño (según Cohen). El metaanálisis más reciente es el de Robertson y cols. ${ }^{27}$, que interesantemente incluyó 70 estudios revisados por los dos anteriores, enfocados en los factores psicosociales vinculados con la DPP ( $\mathrm{n}=14.000)$, así como estudios más recientes y otros que también reportaron los factores no asociados $(\mathrm{n}=10.000)$.

Dentro de los predictores más fuertes están:

- Antecedente personal de depresión: En 2003, Evans y cols. ${ }^{28}$ evaluaron a 106 mujeres chilenas a los seis meses de su postparto, encontrando que $71,42 \%$ con este predictor desarrollaron una DPP; lo destacaron como el principal factor de riesgo. Esto también ha sido reportado como significativo por otros investigadores ${ }^{25,26,29,30} \mathrm{y}$ se ha relacionado con la persistencia de la DPP tras el manejo agudo ${ }^{31}$.

- Sintomatología depresiva o ansiosa durante el embarazo $^{25,26,29,30,32,33}$.

- Pobre apoyo social y familiar $25,26,32,33$.

- Eventos vitales perturbadores ${ }^{25,26}$.

Los factores de riesgo con tamaño del efecto moderado son:

- Estrés cotidiano ${ }^{26,32,33}$.

- Problemas de pareja ${ }^{25,26}$.

- Estrés en el cuidado del bebéé ${ }^{26,33}$.

- Temperamento o conducta del bebé ${ }^{26}$.

- Baja autoestima ${ }^{26}$.

- Disforia postparto ${ }^{26}$.

Por su parte, los predictores con una menor asociación son:

- Ausencia de pareja ${ }^{26}$.

- Dificultades socioeconómicas ${ }^{25,26}$.

- Complicaciones obstétricas ${ }^{25}$.

- Embarazo no deseado o no planeado ${ }^{26}$.

En Chile, Póo y cols. ${ }^{34}$ desarrollaron un estudio correlacional transversal, con información recolectada en ocho meses en una muestra total de 73 mujeres. Destacaron como predictores significativos a la historia personal y en familiares en primer grado de enfermedad mental, la insatisfacción con el cuerpo tras el parto, y la percepción de sobrecarga con el cuidado del bebé. Además, se añaden el consumo de alcohol en el embarazo, la presencia de violencia intrafamiliar, y un pobre vínculo con el padre del último hijo durante el embarazo o con los padres en el postparto. Alvarado y cols. ${ }^{35}$ siguieron a 125 gestantes hasta los dos meses de su postparto, elaborando un modelo causal de DPP que incluyó las siguientes variables por su significancia estadística: 1) Insatisfacción frente a la relación de pareja; 2) edad adolescente en asociación con la depresión antenatal; y 3 ) la interacción entre el bajo apoyo social y el nivel socioeconómico. Dois y cols. ${ }^{36}$ encontraron una asociación directa con el grado de disfuncionalidad familiar (medido por el APGAR familiar). Por su parte, el Ministerio de Salud recomienda evaluar los siguientes factores de riesgo en los controles prenatales: antecedentes familiares de depresión, suicidio, y otros trastornos psiquiátricos (ej. ansiedad, trastornos del ánimo, y asociados al uso de sustancias), ansiedad materna, falta de 
apoyo especialmente de su pareja o no vivir con éste, violencia doméstica y embarazo no deseado ${ }^{15}$.

En 2009, Oppo y cols. ${ }^{37}$ evaluaron la interrelación de los factores de riesgo utilizando un instrumento de tamizaje ampliamente reconocido, el Inventario Revisado de Predictores de Depresión Posparto (PDPI-R), durante diferentes momentos del embarazo y el postparto; observaron una mayor probabilidad de presentar una DPP durante el primer mes del parto en presencia de: ansiedad en el embarazo, estrés en el cuidado del bebé y bajo apoyo psicosocial. Además, reportaron que sufrir una depresión gestacional asociado al antecedente de depresión aumentó de tres a cuatro veces el riesgo de DPP al sexto mes.

Con relación a una mayor severidad o cronificación de la DPP, Horowitz y Goodman ${ }^{16}$ destacaron que los antecedentes de depresión, el pobre apoyo psicosocial y el estrés parental fueron los factores que se asociaron significativamente con la persistencia de sintomatología a los dos años del postparto, encontrando una prevalencia de $30,6 \%$ para ese período. En Chile, Rojas y cols. ${ }^{38}$ reportaron una correlación de mayor gravedad de la sintomatología depresiva según el puntaje de la EDPE, con menor educación, mayor número de embarazos y de hijos y mayor diferencia en edad con el hermano que antecede al bebé. Sin embargo, esta asociación fue leve y no logró confirmarse al controlarlas estadísticamente con otras variables. Sólo el menor nivel educativo y ser multípara mostraron un modelo válido, explicando $5 \%$ de la varianza.

\section{Abordaje}

La eficacia de las intervenciones psicoterapéuticas en el manejo agudo de la DPP ha sido demostrada fuertemente por datos empíricos, recomendándose como tratamiento de primera línea por el menor riesgo de efectos secundarios en el recién nacido, respecto del uso de psicofármacos en mujeres lactantes ${ }^{39}$. La efectividad de la hormonoterapia no ha sido concluyente. Un metaanálisis ${ }^{40}$ señaló que la administración de estrógenos favoreció a mujeres con DPP severa evaluadas a tres meses del tratamiento $(R R=0,30$; IC 95\%; $[0,14-0,66])$, sin embargo, se basaron en una pequeña muestra de estudios lo que dificulta la generalización de estos resultados. El uso de antidepresivos se ha recomendado para la DPP de moderada a severa. En mujeres que mantienen lactancia materna la decisión debe ser individualizada, sopesando los riesgos y beneficios de su administración y brindando la adecuada información a la paciente. En este sentido, se destaca el uso de los inhibidores selectivos de la recaptación de serotonina (ISRS) (especialmente sertralina y paroxetina) por mostrar niveles indetectables en el bebé, a diferencia de la fluoxetina y el citalopram que muestran mayor paso a la leche materna ${ }^{41}$.

En Chile, Rojas y cols. ${ }^{42}$ desarrollaron un ensayo clínico controlado (ECC) en mujeres con DPP $(n=230)$ atendidas en la Atención Primaria de Salud (APS) de sectores vulnerables de Santiago, ingresadas dentro del año de su parto y evaluadas al tercer y sexto mes posterior a la distribución aleatoria. El diagnóstico de DPP se confirmó tras la aplicación de dos EDPE con un par de semanas de diferencia. Si ambas eran positivas con un puntaje de corte de 9/10, se usó la Mini-Entrevista Neuropsiquiátrica Internacional (MINI). Las participantes se asignaron aleatoriamente a una intervención multi-componente (IMC) o a cuidados usuales. La primera incluyó un abordaje psicoterapéutico grupal con elementos psico-educativos y cognitivos-conductuales, monitoreo sistemático de la evolución clínica y adherencia al tratamiento, y farmacoterapia (fluoxetina o sertralina), si era necesaria. La psicoterapia grupal fue la herramienta de primera línea y correspondió a una adaptación de un estudio previo ${ }^{43}$. Sus resultados principales destacaron a la IMC por: 1) Menores puntajes promedios en la EDPE al tercer y sexto mes de medición; 2) mayor reducción en el número de mujeres recibiendo antidepresivos al tercer mes y 3) diferencia significativa en los promedios ajustados de la EDPE al comparar ambos grupos que alcanzó a -4,5 puntos (IC 95\%; [-6,3 a -2,7]; p $<0,0001)$ a los tres meses del seguimiento. Entre los aspectos metodológicos de este estudio se destacan varios puntos: 1) Los investigadores seleccionaron participantes de APS, lo que hace que sus resultados sean especialmente relevantes, pues es donde se atiende la mayoría de la población adscrita a la red pública de salud; 2) se enfocaron en mujeres con bajos ingresos socioeconómicos, factor asociado con mayor prevalencia de DPP; 3 ) se incluyeron mujeres hasta los 12 meses de su postparto, que es el tiempo promedio de permanencia de la sintomatología sin tratamiento y 4) afinaron el diagnóstico de DPP para disminuir falsos positivos.

Con relación a la prevención en DPP, la mayo- 
ría de estos estudios se han realizado en las últimas décadas, incluyendo mujeres con y sin factores de riesgo y usando diseños metodológicos diversos (ej. según tamaños muestrales, abordajes, tipo de profesionales de las intervenciones, etc.) ${ }^{39}$. Varias investigaciones destacan diferencias significativas en los casos de DPP o en los síntomas depresivos postparto tras la implementación de psicoterapia grupal interpersonal (PI $)^{44-47}$ y de psicoterapia cognitivo-conductual (PCC) ${ }^{48-51}$ (Tabla 1). Algunos aspectos relevantes del diseño de estos estudios incluyen: 1) Se enfocaron en la prevención indicada o selectiva; 2) la mayoría son ECC y extendieron el seguimiento de 3 a 12 meses y 3 ) todos estan- darizaron sus desenlaces primarios y secundarios (Tabla 1). Respecto del tipo de intervención, cabe destacar las investigaciones de Muñoz y cols. ${ }^{48}$, Le y cols. ${ }^{50}$ y Tandon y cols. ${ }^{51}$ quienes se basaron en el "Programa de Madres y Bebés" en sus diferentes versiones. Este programa fue desarrollado por Muñoz y cols. (1999) y utiliza la PCC grupal de forma protocolizada. Su objetivo es promover el "manejo saludable de la realidad" a través del incremento de pensamientos y actividades positivas que mejoren el estado de ánimo ${ }^{48,50}$.

Dos metaanálisis ${ }^{52,53}$ basados en estudios preventivos que usaron intervenciones psicosociales y psicológicas mostraron resultados favorables.

Tabla 1. Resumen de Estudios Preventivos

\begin{tabular}{|c|c|c|c|c|c|c|c|}
\hline Autores & Estudio & $\mathbf{n}$ & Selección & $\begin{array}{c}\text { Grupo de } \\
\text { Intervención }\end{array}$ & $\begin{array}{l}\text { Grupo de } \\
\text { Controles }\end{array}$ & $\begin{array}{l}\text { Seguimiento } \\
\text { e Instrumentos }\end{array}$ & $\begin{array}{l}\text { Resultados } \\
\text { principales }\end{array}$ \\
\hline $\begin{array}{l}\text { Zlotnick } \\
\text { y cols. } \\
2001^{44}\end{array}$ & $\begin{array}{c}\text { ECC } \\
\text { (Piloto) }\end{array}$ & 37 & $\begin{array}{l}\text { Factores } \\
\text { de Riesgo }\end{array}$ & $\begin{array}{l}\text { PI grupal prenatal } \\
\text { (4 sesiones) }\end{array}$ & $\begin{array}{c}\text { Cuidados } \\
\text { usuales }\end{array}$ & $\begin{array}{l}3 \text { meses PP; BDI, } \\
\text { SCID-I }\end{array}$ & $\begin{array}{c}\text { Casos de DPP }= \\
0 \%(G \mathrm{l}) \text { vs } 33 \%(\mathrm{GC}) \\
p=0,02\end{array}$ \\
\hline $\begin{array}{l}\text { Zlotnick } \\
\text { y cols. } \\
2006^{45}\end{array}$ & ECC & 99 & $\begin{array}{l}\text { Factores } \\
\text { de Riesgo }\end{array}$ & $\begin{array}{l}\text { PI grupal prenatal } \\
\text { (4 sesiones) y } 1 \\
\text { refuerzo PP }\end{array}$ & $\begin{array}{l}\text { Cuidados } \\
\text { usuales }\end{array}$ & $\begin{array}{c}3 \text { meses PP; } \mathrm{BDI} \text {, } \\
\text { Entrevista diag- } \\
\text { nóstica* }\end{array}$ & $\begin{array}{c}\text { Casos de DPP }= \\
4 \%(\mathrm{Gl}) \text { vs } 20 \%(\mathrm{GC}) \\
p=0,04\end{array}$ \\
\hline $\begin{array}{l}\text { Spinelli } \\
\text { y cols. } \\
1997^{46}\end{array}$ & $\begin{array}{l}\text { Ensayo } \\
\text { Abierto }\end{array}$ & 13 & $\begin{array}{l}\text { Depresión } \\
\text { gestacional }\end{array}$ & $\begin{array}{l}\text { PI pre y postnatal } \\
\text { (16 sesiones) }\end{array}$ & - & $\begin{array}{l}\text { Máx. } 4 \text { meses; } \\
\text { Hamilton+, BDI, } \\
\text { CGI, EDPE }\end{array}$ & $\begin{array}{c}\text { Reducción de EDPE } \\
(p=0,001) ; 10 / 13 \sin \\
\text { síntomas de DPP }\end{array}$ \\
\hline $\begin{array}{l}\text { Spinelli } \\
\text { y cols. } \\
2003^{47}\end{array}$ & ECC & 50 & $\begin{array}{l}\text { Depresión } \\
\text { gestacional }\end{array}$ & $\begin{array}{l}\text { PI pre y postnatal } \\
\text { (16 sesiones) }\end{array}$ & $\begin{array}{l}\text { Psicoe- } \\
\text { ducación } \\
\text { parental }\end{array}$ & $\begin{array}{l}4 \text { meses; Hamil- } \\
\text { ton, BDI, CGI, } \\
\text { EDPE, Escala de } \\
\text { Maudsley** }\end{array}$ & 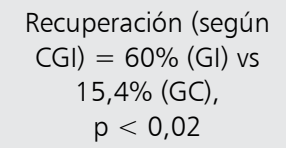 \\
\hline $\begin{array}{l}\text { Muñoz } \\
\text { y cols. } \\
2007^{48}\end{array}$ & $\begin{array}{c}\text { ECC } \\
\text { (Piloto) }\end{array}$ & 41 & $\begin{array}{l}\text { Factores } \\
\text { de Riesgo }\end{array}$ & $\begin{array}{l}\text { PCC grupal prenatal } \\
\text { (12 sesiones) y } 4 \text { de } \\
\text { refuerzos PP }\end{array}$ & $\begin{array}{l}\text { Cuidados } \\
\text { usuales }\end{array}$ & $\begin{array}{c}12 \text { meses; CES-D, } \\
\text { EDPE, MMS }\end{array}$ & $\begin{array}{c}\text { Casos de DPP }=14 \% \\
\begin{array}{c}(G I) \text { vs } 25 \%(G C) \\
h=0,28\end{array}\end{array}$ \\
\hline $\begin{array}{l}\text { Cho } \\
\text { y cols. } \\
2008^{49}\end{array}$ & $\begin{array}{c}\text { ECC } \\
\text { (Piloto) }\end{array}$ & 27 & $\begin{array}{l}\text { Depresión } \\
\text { gestacional }\end{array}$ & $\begin{array}{l}\text { PCC individual pre- } \\
\text { natal (9 sesiones) }\end{array}$ & $\begin{array}{l}\text { Cuidados } \\
\text { usuales }\end{array}$ & $\begin{array}{l}1 \text { mes; BDI, } \\
\text { SCID-I, MSI-R, } \\
\text { ATQ }\end{array}$ & $\begin{array}{c}\mathrm{Gl}=\text { reducción de } \\
\text { síntomas de } \mathrm{DPP}, \\
p<0,01\end{array}$ \\
\hline $\begin{array}{l}\text { Le y cols. } \\
2011^{50}\end{array}$ & ECC & 217 & $\begin{array}{l}\text { Factores } \\
\text { de Riesgo }\end{array}$ & $\begin{array}{l}\text { PCC grupal prenatal } \\
\text { (8 sesiones) y } 3 \text { de } \\
\text { refuerzos PP }\end{array}$ & $\begin{array}{l}\text { Cuidados } \\
\text { usuales }\end{array}$ & $\begin{array}{c}12 \text { meses; CES-D, } \\
\text { EDPE, BDI-II, } \\
\text { MMS }\end{array}$ & $\begin{array}{l}\mathrm{Gl}=\text { reducción de } \mathrm{BDI}- \\
\text { II post-intervención, } \\
\mathrm{p}=0,03\end{array}$ \\
\hline $\begin{array}{l}\text { Tandon } \\
\text { y cols. } \\
2011^{51}\end{array}$ & ECC & 61 & $\begin{array}{l}\text { Factores } \\
\text { de Riesgo }\end{array}$ & $\begin{array}{l}\text { PCC grupal prenatal } \\
\text { (6 sesiones) y visitas } \\
\text { de refuerzo } 1 / 1\end{array}$ & $\begin{array}{l}\text { Cuidados } \\
\text { usuales }\end{array}$ & $\begin{array}{c}3 \text { meses; BDI-II, } \\
\text { MMS }\end{array}$ & $\begin{array}{c}\text { Casos de DPP }=9 \% \\
(G I) \text { vs } 33 \%(G C) \\
p<0,05\end{array}$ \\
\hline
\end{tabular}

$\mathrm{ECC}=$ Ensayo Clínico Controlado; $\mathrm{PI}=$ Psicoterapia Interpersonal; $\mathrm{PCC}=$ Psicoterapia Cognitivo-Conductual; $\mathrm{PP}=\mathrm{Postparto;}$ $\mathrm{BDI}=$ Escala de Depresión de Beck; BDI-II=Escala de Depresión de Beck segunda versión; SCID-I= Entrevista Clínica Estructurada para los Trastornos del Eje I del DSM-IV; Escala de Depresión de Hamilton+; CGI= Escala de Impresión Clínica Global; $E D P E=$ Escala de Depresión Postparto de Edimburgo; CES-D = Escala de Depresión del Centro de Estudios Epidemiológicos; MMS = Maternal Mood Screener; MSI-R = Inventario de Satisfacción Marital de Snyder Revisado; ATQ= Cuestionario de Pensamientos Automáticos; Longitudinal Interval Follow-up Evaluation (Depression Module)*; The Maudsley Mother Infant Interaction Scale** $\mathrm{DPP}=$ Depresión Postparto; GI = Grupo de Intervención; GC= Grupo de Controles. 
El primero evaluó 14 estudios en mujeres con bajo nivel socioeconómico $(\mathrm{n}=1.396)$, reportando un tamaño del efecto de 0,31 , que fue considerado como significativo (IC 95\%; $[0,17-0,45])^{52}$. El segundo incluyó 28 estudios $(\mathrm{n}=17.000)$ y destacó lo siguiente: 1) La importancia de detectar factores de riesgo para favorecer la prevención $(\mathrm{RR}=0,66$; IC 95\%; [0,50-0,88]);2) el impacto positivo de las intervenciones realizadas por profesionales (ej. PI o visitas domiciliarias por enfermeras o matronas) y por otros (ej. soporte telefónico por consejeros); 3) la utilidad de los abordajes postnatales en reducir síntomas depresivos $(\mathrm{RR}=0,73$; IC 95\%; $[0,59-0,90] ; \mathrm{n}=12.786)$ por sobre los que incluyeron ambos componentes $(\mathrm{RR}=0,96$; IC $95 \%$; $[0,75-1,22] ; \mathrm{n}=1.941)$ y 4 ) mayor beneficio de las intervenciones individuales que de las grupales $(\mathrm{RR}=0,75 \text {; IC 95\%; [0,61-0,92] })^{53}$.

\section{Discusión y Conclusiones}

Es ampliamente conocido que las mujeres presentan una mayor prevalencia de trastornos depresivos y que el riesgo de desarrollarlos puede aumentar en ciertos momentos de su vida. El postparto representa un período de mayor susceptibilidad en vista de las fluctuaciones del eje hormonal, las demandas propias del cuidado del recién nacido y la interacción de variables psicosociales. Aunque es claro el desafío fisiológico enfrentado en el postparto, el peso de las variables biológicas como causa de la DPP no ha podido demostrarse de forma consistente, a diferencia de otros trastornos psiquiátricos perinatales como la psicosis postparto. Una eventual explicación de esta pérdida de asociación es la gran confluencia de otras variables, como las psicosociales, pero además se han reportado limitaciones en la correlación entre los niveles hormonales centrales y periféricos ${ }^{40}$.

La génesis de la DPP también genera discusiones frente al impacto del "determinismo socio-cultural", pues es evidente que la forma en que las distintas sociedades asumen la gestación, el nacimiento, la transición de roles y la capacidad de las redes de apoyo de articularse en torno a estos acontecimientos, tendrá relación con el devenir de cada postparto. Lo cierto es que las expectativas asociadas al nivel de perfección con que debe asumirse el rol materno pueden convertirse en un estresor adicional y ser un obstáculo para buscar una atención adecuada. Este punto es especialmente relevante, pues el temor a ser devaluada o criticada puede interferir con la búsqueda de ayuda oportuna en una mujer cursando con síntomas depresivos pre o post-parto.

En este sentido, un abordaje apropiado de la DPP exige un mayor reconocimiento de los aspectos psicológicos en toda gestante durante sus controles prenatales pues, como ya se ha expuesto, la depresión antenatal y el historial depresivo son predictores fuertemente asociados con su ocurrencia.

Un mayor énfasis en la salud mental perinatal $\mathrm{y}$ en los factores de riesgo que pueden comprometerla es una de las estrategias más simples de prevención si se implementa de forma sistemática durante el seguimiento. La aplicación de instrumentos de tamizaje como la EDPE es una estrategia útil y sencilla para precisar la sospecha de una DPP y su uso en Chile está bien justificado por lo siguiente: 1) Ha sido validada adecuadamente ${ }^{21}$; 2) el Ministerio de Salud ha recomendado su aplicación durante el embarazo y el postparto ${ }^{15} \mathrm{y}$ 3) la evidencia ha arrojado aumento en las tasas de detección de síntomas de DPP en comparación con el reporte espontáneo ${ }^{22}$.

Respecto del abordaje de la DPP, la literatura destaca las intervenciones psicoterapéuticas como primera línea de tratamiento. La administración de antidepresivos en mujeres lactantes debe evaluarse en cada caso, considerando los riesgos y ventajas, destacándose el uso de algunos ISRS por su menor detección en la leche. La presente revisión se ha enfocado en las intervenciones preventivas, teniendo en cuenta que hay mayor consenso y experiencia respecto de su tratamiento que de la prevención. La evaluación de la información recolectada nos permite recomendar la implementación de intervenciones preventivas de corte psicosocial en mujeres de alto riesgo, no sólo por la mejoría de los síntomas depresivos o en la incidencia de DPP, sino porque optimizar la salud mental de la mujer durante su embarazo y postparto beneficiará al bebé en términos de una mejor interacción de la díada, del desarrollo de vínculos de apego seguro y en su bienestar global $^{19,53}$. Existe adecuada evidencia nacional ${ }^{42,43}$ respecto del tratamiento de la DPP, pero escasa respecto de su prevención. Se requieren estudios nacionales que evalúen la efectividad de estas intervenciones. 


\section{Referencias}

1. Wisner KL, Perel JM, Peindl KS, Hanusa BH, Piontek CM, Findling RL. Prevention of Postpartum Depression: A Pilot Randomized Clinical Trial. Am J Psychiatry 2004; 161: 1290-2.

2. Asociación Americana de Psiquiatría. Manual Diagnóstico y Estadístico de los Trastornos Mentales, quinta edición (DSM-V). Arlington-EE.UU., 2013. Disponible en: www.dsm.psychiatryonline.org. [Consultado en julio de 2014].

3. Asociación Americana de Psiquiatría. Manual Diagnóstico y Estadístico de los Trastornos Mentales, cuarta edición revisada. Breviario (DSM-IV-TR). Barcelona, España: Editorial Masson S.A.; 2003. p. 191-2.

4. Wisner KL, Parry BL, Piontek CM. Clinical Practice. Postpartum depression. N Engl J Med 2002; 347: 194-9.

5. Josefsson A, Berg G, Nordin C, Sydsjô G. Prevalence of depressive symptoms in late pregnancy and postpartum. Acta Obstet Gynecol Scand 2001; 80 (3): 251-5.

6. Troutman B, Cutrona CE. Nonpsychotic postpartum depression among adolescent mothers. Journal of Abnormal Psychology 1990; 99: 69-78.

7. Hobfoll SE, Ritter C, Lavin J, Hulsizer MR, Cameron RP. Depression prevalence and incidence among inner-city pregnant and postpartum woman. Journal of Consulting and Clinical Psychology 1995; 63 (3): 44553.

8. Lee DTS, Yip ASK, Chiu HFK, Leung TYS, Chung KH. A Psychiatric Epidemiological Study of Postpartum Chinese Women. Am J Psychiatry 2001; 158: 220-6.

9. Patel V, De Souza N, Rodrígues M. Postnatal depression and infant growth and development in low income countries: a cohort study from Goa, India. Arch Dis Child 2002; 87: 1-4.

10. Alvarado R, Rojas M, Monardes J, Neves E, Olea E, Perucca E, et al. Cuadros depresivos en el postparto y variables asociadas en una cohorte de 125 mujeres embarazadas. Rev Psiquiatr 1992; 3: 1168-76.

11. Jadresic E, Jara C, Miranda M, Arrau B, Araya R. Trastornos emocionales en el embarazo y el puerperio: estudio prospectivo de 108 mujeres. Rev Chil Neuro-Psiquiat 1992; 30: 99-106.

12. Jadresic E, Araya R. Prevalencia de depresión postparto y factores asociados en Santiago, Chile. Rev Med Chile 1995; 123: 694-9.

13. Gregoire AJ, Kumar R, Everitt B, Henderson AF, Studd JW. Transdermal oestrogen for treatment of severe postnatal depression. Lancet 1996; 347: 930-3.

14. Cooper PJ, Murray L. Course and recurrence of postnatal depression. Evidence for the specificity of the diagnostic concept. Br J Psychiatry 1995; 166 (2): 191-5.

15. Ministerio de Salud-Gobierno de Chile. Subsecretaría de Salud Pública. Protocolo de detección de la depresión durante el embarazo y postparto, y apoyo al tratamiento. Santiago, Chile. 2014.

16. Horowitz JA, Goodman J. A Longitudinal Study of Maternal Postpartum Depression Symptoms. Research and Theory for Nursing Practice: An International Journal 2004; 18 (2/3): 149-63.

17. Paris R, Bolton RE, Weinberg MK. Postpartum depression, suicidality, and mother-infant interactions. Arch Women Mental Health 2009; 12: 309-21.

18. Ministerio de Salud-Gobierno de Chile. Guía Clínica: Tratamiento de Personas con Depresión. Santiago, Chile. 2009.

19. Cooper PJ, Murray L. Fortnightly review: Postnatal depresssion. BMJ 1998; 316: 1884-6.

20. Deater-Deckard K, Pickering K, Dunn JF, Golding J. Family Structure and Depressive Symptoms in Men Preceding and Following the Birth of a Child. Am J of Psychiatry 1998; 155: 818-23.

21. Jadresic E, Araya R, Jara C. Validation of the Edinburgh postnatal depression scale (EPDS) in Chilean postpartum women. J Psychosom Obstet Gynecol 1995; 16: 187-91.

22. Castañón C, Pinto J. Mejorando la pesquisa de depresión posparto a través de un instrumento de tamizaje, la escala de depresión posparto de Edimburgo. Rev Med Chile 2008; 136: 851-8.

23. Evins GG, Theofrastous JP, Galvin SL. Postpartum depression: A comparison of screening and routine clinical evaluation. Am J Obstet Gynecol 2000; 182: 1080-2.

24. Jadresic E. Depresión Postparto: Aspectos Antropológicos y Transculturales. Psykhe 1996; 5 (2): 149-57.

25. O'Hara MW, Swain AM. Rates and risk of postpartum depression-a meta-analysis. Int Rev Psychiatry 1996; 8: 37-54.

26. Beck CT. Predictors of Postpartum Depression: an update. Nurs Res 2001; 50 (5): 275-85.

27. Robertson E, Sherry G, Wallington T, Stewart DE. Antenatal risk factors for postpartum depression: a synthesis of recent literature. Gen Hosp Psychiatry 2004; 26: 28995.

28. Evans G, Vicuña M, Marín R. Depresión Postparto Realidad en el Sistema Público de Atención de Salud. Rev Chil Obstet Ginecol 2003; 68 (6): 491-4.

29. Josefsson A, Angelsioo L, Berg G, Ekstrom CM, Gunnervick C, Nordin C, et al. Obstetric, somatic, and demographic risk factors for postpartum depressive symptoms. Obstet Gynecol 2002; 99: 223-8.

30. Johnstone SJ, Boyce PM, Hickey AR, Morris-Yatees AD, 
Harris MG. Obstetric risk factors for postnatal depression in urban and rural community samples. Aust NZJ Psychiatry 2001; 35: 69-74.

31. Nylen KJ, O’Hara MW, Brock R, Moel J, Gorman L, Stuart S. Predictors of the longitudinal course of postpartum depression following interpersonal psychotherapy. Journal of consulting and clinical psychology 2010; 78 (5): 757-63.

32. Aramburú P, Arellano R, Jáuregui S, Pari L, Salazar P, Sierra O. Prevalencia y factores asociados a depresión posparto en mujeres atendidas en establecimientos de salud del primer nivel de atención en Lima Metropolitana. Revista Peruana de Epidemiología 2008; 12 (3): 1-5.

33. Hatton DC, Hohner-Harrison J, Matarazzo J, Edwards P, Lewy A, Davis L. Missed antenatal depression among high risk women: a secondary analysis. Arch Women's Ment Health 2007; 10: 121-31.

34. Póo AM, Espejo C, Godoy C, Gualda de la CM, Hernández T, Pérez C. Prevalencia y factores de riesgo asociados a la depresión postparto en puérperas de un Centro de Atención Primaria en el Sur de Chile. Rev Med Chile 2008; 136: 44-52.

35. Alvarado R, Rojas M, Monardes J, Perucca E, Neves E, Olea E, et al. Cuadros depresivos en el postparto en una cohorte de embarazadas: construcción de un modelo causal. Rev Chil Neuro-Psiquiat 2000; 38 (2): 84-93.

36. Dois A, Uribe C, Villarroel L, Contreras A. Factores de riesgo asociados a síntomas depresivos post-parto en mujeres de bajo riesgo obstétrico atendidas en el sistema público. Rev Med Chile 2012; 140: 719-25.

37. Oppo A, Mauri M, Ramacciotti D, Camilleri V, Banti S, Borri C, et al. Risk factors for postpartum depression: the role of the Postpartum Depression Predictors Inventory-Revised (PDPI-R). Arch Women's Ment Health 2009; 12: 239-49.

38. Rojas G, Fritsch R, Guajardo V, Rojas F, Barroilhet S, Jadresic E. Caracterización de madres deprimidas en el postparto. Rev Med Chile 2010; 138: 536-42.

39. Stuart S, O`Hara MW, Gorman LL. The prevention and psychotherapeutic treatment of postpartum depression. Arch Women's Ment Health 2003; 6 (Suppl. 2): s57-s69.

40. Dennis CL, Ross LE, Herxheimer A. Oesstrogens and progestins for preventing and treating postpartum depression (Review). The Cochrane Lybrary 2010; 10: 1-23. Disponible en: www.thecochranelibrary.com. [Consultado en el segundo semestre de 2014].

41. Fitelston E, Kim S, Scott A, Leight K. Treatment of postpartum depression: clinical, psychological and pharmacological options. Internation Journal of Women's Health 2011; 3: 1-14.
42. Rojas G, Fritsch R, Solís J, Jadresic E, Castillo C, González $\mathrm{M}$, et al. Treatment of postnatal depression in low-income mothers in primary-care clinics in Santiago, Chile: a randomized controlled trial. The Lancet 2007; 370: 1629-37.

43. Araya R, Rojas G, Fritsch R, Gaete J, Rojas M, Simon G, et al. Treating depression in primary care in low-income in Santiago, Chile. The Lancet 2003; 361: 995-1000.

44. Zlotnick C, Johnson SL, Miller IW, Pearlstein T, Howard M. Postpartum depression in women receiving public assistance: pilot study of an interpersonal-therapy-oriented group intervention. Am J Psychiatry 2001; 158: 638-40.

45. Zlotnick C, Miller IW, Pearlstein T, Howard M, Sweeney P. A Preventive Intervention for Pregnant Women on Public Assistance at Risk for Postpartum Depression. Am J Psychiatry 2006; 163: 1443-5.

46. Spinelli MG. Interpersonal Psychotherapy for Depressed Antepartum Women: A Pilot Study. Am J Psychiatry 1997; 154: 1028-30.

47. Spinelli MG, Endicott J. Controlled Clinical Trial of Interpersonal Psychotherapy Versus Parenting Education Program for Depressed Pregnant Women. Am J Psychiatry 2003; 160; 555-62.

48. Muñoz RF, Le HN, Ghosh Ippen CH, Díaz MA, Urizar Jr. GG, Soto J, et al. Prevention of Postpartum Depression in Low-Income Women: Development of the Mamás y Bebés/Mothers and Babies Course. Cognitive and Behavioral Practice 2007; 14: 70-83.

49. Cho HJ, Kwon JH, Lee JJ. Antenatal Cognitive-Behavior Therapy for Prevention of Postpartum Depression: A Pilot Study. Yonsei Med J 2008; 49 (4): 553-62.

50. Le HN, Perry DF, Stuart EA. Randomized Controlled Trial of a Preventive Intervention for Perinatal Depression in High Risk Latinas. Journal of Consulting and Clinical Psychology 2011; 79: 131-45.

51. Tandon D, Perry D, Mendelson T, Kemp K, Leis JA. Preventing Perinatal Depression in Low-Income Home Visiting Clients: A randomized controlled trial. Journal of Consulting and Clinical Psychology 2011; 79 (5): 70712.

52. Van der Waerden J, Hoefnagels C, Hosman C. Psychosocial preventive interventions to reduce depressive symptoms in low-SES women at risk: A meta-analysis. Journal of Affective Disorders 2011; 128: 10-23.

53. Dennis CL, Dowswell T. Psychosocial and psychological interventions for prevention of postnatal depression (Systematic Review). The Cochrane Library 2013; 2: 1-151. Disponible en: www.thecochranelibrary.com. [Consultado en el segundo semestre de 2014]. 